\title{
REFERENCES
}

1. R. Hargreaves, A pressure-integral as kinetic potential, Phil. Mag. 436-444 (1908).

2. H. Bateman, Notes on a differential equation which occurs in the two-dimensional motion of a compressible fluid and the associated variational problems, Proc. Roy. Soc. London (A) 125, 598-618 (1929).

3. Chi-Teh Wang, Variational method in the theory of compressible fluid, J. of Aero. Sciences 15, 675-685 (1948).

4. Chi-Teh Wang, Two dimensional subsonic compressible flows past arbitrary bodies by the variational method. Report submitted to NACA, June 1949.

5. Chi-Teh Wang and G. V. R. Rao, A study of the non-linear characteristic of compressible flow equations by means of variational method, J of Aero. Sciences 17, 343-348 (1950).

6. Chi-Teh Wang and R. F. Brodsky, Application of Galerkin's method to compressible fluid flow problems, J. Appl. Phys. 20, 1255-1256 (1949).

7. Chi-Teh Wang and R. F. Brodsky, Approximate solution of compressible fluid flow problems by Galerkin's method, J. of Aero Sciences 17, 660-666 (1950).

8. Chi-Teh Wang and Pei-Chi Chou, Application of Biezieno-Koch method to compressible fluid flow problems, J. of Aero Sciences 17, 599-660 (1950).

9. Chi-Teh Wang and S. de los Santos, Approximate solutions of compressible flows past bodies of revolution by variational method, to be published in J. of Appl. Mech. as Paper No. 50-A-33.

\section{ON A GEOMETRICAL METHOD OF DERIVING THREE-DIMENSIONAL HARMONIC FLOWS FROM TWO-DIMENSIONAL ONES*}

\section{BY AUREL WINTNER (The Johns Hopkins University)}

The Flow Operators $\Omega$. Let $D$ be a domain in a $(u, v)$-plane, $E$ a domain in an $(x, y, z)$ space, and let $\varphi=\varphi(u, v), \psi=\psi(x, y, z)$ denote (real-valued and regular) solutions of $\Delta_{2} \varphi=0, \Delta_{3} \psi=0$ on $D, E$, respectively, where $\Delta_{2}$ and $\Delta_{3}$ denote the two- and threedimensional euclidean Laplace operators,

$$
\Delta_{2}=\partial^{2} / \partial u^{2}+\partial^{2} / \partial v^{2} \text { and } \Delta_{3}=\partial^{2} / \partial x^{2}+\partial^{2} / \partial y^{2}+\partial^{2} / \partial z^{2} .
$$

All harmonic functions $\varphi$ are accessible in principle, since all of them are given by $\varphi(u, v)=\operatorname{Re\chi }(w)$, where $\chi$ is any function which is regular-analytic in $w=u+i v$ on $D$. In contrast, there does not exist anything like this rule for the harmonic functions $\psi(x, y, z)$ on a three-dimensional $E$. Hence it is natural to ask for flow operators, say $\Omega=\Omega(D)$, which, from every regular solution $\varphi=\varphi(u, v)$ of $\Delta_{2} \varphi=0$ on a two-dimensional $(u, v)$-domain $D$, will manufacture a regular solution,

$$
\psi(x, y, z)=\Omega \varphi(u, v),
$$

of $\Delta_{3} \psi=0$ on a three-dimensional $(x, y, z)$-domain $E=E(D)$. The latter should not depend on the particular choice of the function $\varphi(u, v)$, but merely on the operator $\Omega=\Omega(D)$ and on the domain $D$ on which $\varphi(u, v)$ is supposed to be harmonic.

A trivial instance of such "harmonic flow operators" $\Omega$ is supplied by the cylindrical flow which, from a given $\varphi(u, v)$, manufactures the corresponding $\psi(x, y, z)$ as follows:

$$
\psi(x, y, z)=\varphi(x, y) .
$$

In fact, (3) is of the type (2), since $\Delta_{3} \psi(x, y, z)=\Delta_{2} \psi(x, y)$ if $\partial^{2} \psi / \partial z^{2}=0$; cf. (1). The

*Received June 30, 1950. 
$E=E(D)$ belonging to the case (3) of (2) is the interior of the infinite cylinder having the (orthogonal) cross-section $D$.

The History of the Problem. It turns out that this trivial flow operator $\Omega$, that defined by (3), is only the simplest instance of a large class of flow operators $\Omega$ which have the property specified in connection with (2). In fact, the general class of harmonic flow operators $\Omega$ was discovered by Weingarten sixty years ago. ${ }^{1}$ His results are stated, however, in the language of the differential geometry of minimum surfaces, and seem to have remained unnoticed (except, possibly, ${ }^{2}$ by F. Klein). In any case, the principal result of Weingarten was rediscovered by Levi-Civita a decade later, ${ }^{3}$ by using, instead of the theory of minimum surfaces, extremely heavy explicit calculations, which he only was able to carry out due to Ricci's and his absolute differential calculus. In addition, these results of Levi-Civita are in a section which is not along the main lines of his long paper. Finally, the latter was published in a periodical not available in most libraries.

These circumstances notwithstanding, it is somewhat surprising that the $\Omega$-flows in question seem to have been forgotten for half a century. On the other hand, the nature and the generality of the result appear to be elastic enough to admit its explicit use in solving certain three-dimensional boundary-value problems which could hardly be attacked in any other way. Under these circumstances, and because only the proof, but not the final result, is quite involved, the criterion in question seems to be worth recording here in a form which, it is hoped, can be followed by those who apply mathematics.

(i) Line Congruences. Let $\Gamma$ be a 2-parameter family of straight lines which, with reference to a three-dimensional $(x, y, z$,$) -domain E$, have the following property: If $P$ is any point of $E$, then $\Gamma$ contains exactly one straight line, say $\gamma=\gamma_{P}$, through $P$. In the terminology of the differential geometry of "rays" $\gamma$, such a collection $\Gamma$ is called a "line congruence" (on $E$ ). It will be assumed that that portion of each of the straight lines which is contained in $E$ is a connected set (i.e., that it is a segment or a half-line, possibly the entire line $\gamma$ ).

Analytically, every sufficiently small portion of $\Gamma$ can be described by first choosing inside $E$ a surface, say $S$, and then introducing on $S$ Gaussian parameters, say $u$ and $v$, in an arbitrary way, subject only to the restriction that there be a one-to-one (and sufficiently differentiable) correspondence between the points of $S$ and the points of a domain, say $D$, in a euclidean $(u, v)$-plane. In fact, if $S$ and its $(u, v)$-parametrization are fixed, then a straight line $\gamma$ belonging to $\Gamma$ can be individualized by placing $\gamma=$ $\gamma(u, v)$, where $u, v$ are the parameter values of that point of $S$ from which $\gamma$ is issued.

(ii) Rectilinear Flows. In this manner, the line congruence $\Gamma$ defines a rectilinear flow from the surface $S$ into the three-dimensional $(x, y, z)$-domain $E$ (which contains $S$ ).

1J. Weingarten, Ueber particuläre Integrale der Differentialgleichung $\Delta V=0$ und eine mit der Theorie der Minimalflächen zusammenhängende Gattung von Flüssigkeitsbewegungen, Nachrichten von der Königlichen Gesellschaft der Wissenschaften zu Göttingen, 1890, pp. 313-335.

${ }^{2} \mathrm{Cf}$. the footnote on p. 117 of Levi-Civita's paper, referred to in the next footnote. The common element in the treatments of Weingarten and Levi-Civita is Jacobi's theorem concerning the (complexvalued) solutions $f=f(x, y, z)$ of $(\partial f / \partial x)^{2}+(\partial f / \partial y)^{2}+(\partial f / \partial z)^{2}=0$, a theorem to which Levi-Civita's attention was called by Klein (cf. the footnote mentioned before).

${ }^{3}$ T. Levi-Civita. Tipi di potenziali che si possono far dipendere da due sole coordinate, Memorie della Reale Accademia delle Scienze de Torino, ser. 2, vol. 49 (1900), pp. 105-152; more particularly, pp. 138-139. 
Correspondingly, if $\varphi$ is any function of the position $(u, v)$ on a two-dimensional domain, $D$, in the euclidean $(u, v)$-plane, with $u, v$ as Gaussian parameters on $S$, then a function, say $\psi$, of the position $(x, y, z)$ on $E$ will be defined by placing

$$
\psi(x, y, z)=\varphi(u, v) \text { if }(x, y, z) \text { is on } \gamma(u, v) .
$$

It is understood that $\gamma(u, v)$ in (4) denotes that straight line, $\gamma$, of $\Gamma$ which reaches the surface $S$ at the point having the Gaussian coordinates $(u, v)$.

(iii) Laplacean Rectilinear Flows. Clearly, the operation (4) is of the type (2). But it was not assumed in (4) that the given function, $\varphi(u, v)$, satisfies $\Delta_{2} \varphi=0$. Moreover, if $\Delta_{2} \varphi=0$ is assumed, it is not in general true that the function $\psi(x, y, z)$ defined by (4) will satisfy $\Delta_{3} \psi=0$. The operators $\Delta_{2}, \Delta_{3}$ are those defined by (1).

This problem is solved by the result of Weingarten and Levi-Civita, referred to above. It states that, if $\varphi(u, v)$ is any (continuous) solution of $\Delta_{2} \varphi=0$ on a $(u, v)$-domain $D$, then the function $\psi(x, y, z)$ defined by (4) will be a solution of $\Delta_{3} \psi=0$ on the corresponding $(x, y, z)$-domain $E=E_{D}$, provided that the rectilinear flow, on which the assignment (4) is based, is derived from a line congruence $\Gamma$ which is an isotropic line congruence.

The latter notion, to be defined under (iv) below, depends only on the collection of lines which constitute $\Gamma$, rather than on the particular choice of the surface $S$ and of the Gaussian parametrization $(u, v)$ of $S$. Consequently, neither of these choices matters, even though both of them occur in (4).

(iv) Isotropy. There remains to be defined the notion of an isotropic line congruence, mentioned above. The customary definition of this notion, introduced by Ribacour, ${ }^{4}$ is quite involved. In what follows, it will be replaced by another definition, one which can be stated more easily and which, as a matter of proof, ${ }^{5}$ turns out to be equivalent to the usual definition.

Consider in the $(x, y, z)$-space two (sufficiently small and sufficiently differentiable) pieces of surfaces, say $S$ and $T$, and let $P \rightarrow Q=Q_{P}$ be a one-to-one (and sufficiently differentiable) mapping of the points, $P$, of $S$ on the points, $Q$, of $T$. Suppose that every $S$-point, $P$, is distinct from its $T$-image, $Q=Q_{P}$, i.e., that every pair $P, Q$ determines a straight line $\gamma=\gamma(P ; Q)$ and therefore the entire mapping, $S \rightarrow T$, defines a certain line congruence, say $\Gamma$. Suppose further that the mapping $S \rightarrow T$ defining this $\Gamma$ has the following two properties:

( $\alpha) P \rightarrow Q=Q_{P}$ is an isometric mapping of $S$ on $T$ (as to this notion, cf. (v) below) and

( $\beta)|P Q|=$ const., i.e., the euclidean length of the segment joining $P$ with $Q=Q_{P}$ (in the $(x, y, z)$-space) is independent of the choice of $P$ on $S$.

If these conditions are satisfied by the mapping $S \rightarrow T$, then the line congruence $\Gamma$, mentioned before $(\alpha)$, is an isotropic line congruence. Conversely, every isotropic line congruence can be obtained in this manner, that is, by suitably choosing two surfaces, $S$ and $T$, and a mapping, $S \rightarrow T$, which satisfies both of the above conditions, $(\alpha)$ and $(\beta)$.

(v) Isometry. For the sake of completeness, the notion of isometry, occurring above in $(\alpha)$, remains to be explained. This notion is familiar from the elements of the theory of surfaces. It is defined as follows:

${ }^{4}$ L. Bianchi, Lezioni di geometria differenziale, 2nd edition, vol. 1 (1902), p. 302.

${ }^{5}$ Loc. cit., ${ }^{4}$ p. 304. 
A one-to-one mapping of the points, $P$, of the surface, $S$, on the points, $Q=Q_{P}$, of another surface, $T$, is called isometric if, by virtue of the mapping, the squared lineelement, $d s^{2}$, on $S$ (i.e., the so-called "metric" or "first fundamental form", $g_{i k} d u^{i} d u^{k}$, on $S$ ) coincides with the squared line-element on $T$.

In order that this be the case, it is necessary that the Gaussian curvature of $S$ at any point $P$ be identical with the Gaussian curvature of $T$ at the corresponding point $Q=Q_{P}$. This necessary condition is sufficient as well in the particular case of surfaces of constant Gaussian curvature. Since cylinders are of constant Gaussian curvature, the constant being 0 , this embeds the trivial example (3) into the general theory.

\section{FREE LONGITUDINAL VIBRATION OF A PROLATE ELLIPSOID, CLAMPED CENTRALLY*}

By JAMES S. KOUVELITES (Sloane Physics Laboratory, Yale University**)

Introduction. It was originally shown by Poisson ${ }^{1}$ that the magnetization is uniform and parallel throughout the interior of an ellipsoid of magnetically isotropic material placed in a previously uniform and parallel static field. Since this unique property of the ellipsoid allows an exact calculation of its demagnetizing factor as well as a simple field analysis, the prolate spheroid was chosen as the appropriate shape for the specimens whose magnetostrictive vibrational properties are being studied in this laboratory. ${ }^{2}$ Although the magnetostrictive vibration is forced rather than free, the damping factor has been found to be so small for the materials tested, that through an investigation of the free vibration, a reasonably good approximation to some of the resonance phenomena may be obtained.

Analysis and discussion. For later comparison with the corresponding expressions for the ellipsoid, the differential equation of motion, its integrated solution, and a subsidiary expression for the frequencies of resonance for free longitudinal vibration of a bar of constant cross-section, clamped centrally, ${ }^{3}$ are

$$
\begin{gathered}
\frac{\partial^{2} \xi}{\partial x^{2}}=\frac{\rho}{k} \frac{\partial^{2} \xi}{\partial t^{2}} \\
\xi=A \sin \left(\omega\left(\frac{\rho}{k}\right)^{1 / 2} x\right) \sin (\omega t+\phi) \\
f_{R_{n}}=\frac{n}{4 a}\left(\frac{k}{\rho}\right)^{1 / 2}, \quad n=1,3,5, \cdots
\end{gathered}
$$

*Received Sept. 26, 1950.

**Assisted by the Office of Naval Research.

${ }^{1}$ As described in J. C. Maxwell, A treatise on electricity and magnetism, vol. II, 3rd ed., Clarendon Press, Oxford, 1892, pp. 66-69.

${ }^{2}$ J. S. Kouvelites and L. W. McKeehan, Magnetostrictive vibration of prolate spheroids. Preliminary measurements, Rev. Sci. Instr., in press.

${ }^{3}$ Rayleigh, Theory of sound, vol. I, \$150. 